\title{
Sequence Time Domain Reflectometry for Transmission Line Analysis
}

\author{
J. Reis, A.L.S. Castro, J.C.W.A Costa, J.R.I. Riu, and K. Ericson
}

\begin{abstract}
This paper describes Sequence Time Domain Reflectometry (STDR), which utilizes concepts from direct sequence spread spectrum communications, as a technique for detecting impedance mismatches in telephone lines (twisted pair). The aim of this paper is to present methodologies for characterizing a subscriber loop, which is used for Digital Subscriber Line (DSL) technology, based on STDR tests. Those tests enable the TDR functionality to be incorporated into a DSL transceiver integrated circuit eliminating the need for costly test equipment. In addition to the cost savings, the characteristics of the STDR offer improved spectral compatibility, interference immunity and fault resolvability.
\end{abstract}

Index Terms-Loop qualification, digital subscriber line, sequence time domain reflectometry, spread spectrum.

\section{INTRODUCTION}

Reflectometry methods have been used for locating faults (impedance mismatches) on wires for decades. These methods send a high frequency signal down the line, which reflects back at impedance mismatches such as open or short circuits, gauge changes and specially in bridged taps [1]. The difference (time delay) between the incident and reflected signal is used to locate the impedance discontinuity on the wire. The nature of the input signal is used to classify each type of reflectometry test. Time Domain Reflectometry (TDR) uses a fast rise time pulse [1], [2], [3]. Frequency Domain Reflectometry (FDR) including Phase Detection Reflectometry (PD-FDR), and Mixed Signal Reflectometry (MSR), uses sinewave signals to locate the fault on the wire. Multicarrier Reflectometry (MCR) uses a combination of sine waves with random phases [4]. Sequence Time Domain Reflectometry (STDR) uses a pseudo-noise sequence (PN sequence), and Spread Spectrum Time Domain Reflectometry (SSTDR) uses a sine-wave-modulated PN sequence [4], [5].

Some traditional reflectometry methods should not be applied under specific conditions, e.g. very short loops $(\leq 200 \mathrm{~m})$ or noisy environment. Besides, those techniques may not detect the impedance discontinuity or detect it with low accuracy. Additionally, it has been shown that traditional reflectometry methods are not effective for location of small fault (which generates weak reflections such as gauge change or far discontinuity) on telephony lines for instance [1]; the reason for that is the returned reflections are smaller than the reflections that are generated from others impedance

J. Reis and J.C.W.A Costa are with Applied Electromagnetism Laboratory, Federal University of Pará, DEEC, CEP: 66075-900, Belém-PA, Brazil. A.L.S. Castro is with the Signal Processing Laboratory, Federal University of Pará, DEEC, CEP: 66075-900, Belém-PA, Brazil. J.R.I. Riu, and K. Ericson are with Ericsson AB, Älvsjö, Sweden. This work was supported by the Research and Development Centre, Ericsson Telecomunicações S.A., Brazil. E-mails: jacklyn, agcastro, jweyl@ufpa.br; jaume.rius.i.riu, klas.ericson@ericsson.com discontinuities in the line such as bridged taps, open and short circuits. Besides, the subscriber telephone loop exhibits distributed RLC behavior which causes the slowly decaying in the test signal. If the reflection is very strong, this behavior may be neglected, whereas in the case of weak reflections, this approximation turns out to be too harsh [1]. Another difficulty in detecting weak reflection lies on the fact that there are spurious reflections and/or noise which interferes in the reflection signal of interest (used for locating faults); then making difficult the location of the discontinuity. It is worth emphasizing that the shorter is the telephony loop, the higher frequency should be used in the test pulse to provide high accurate result for wiring fault location.

The main goal of this paper is to provide better results with high accuracy, for fault location in the subscriber telephone loop than the ones provided by traditional reflectometry techniques by using sequence TDR. Gauge changes, bridged taps as well as total loop length are the main variables that will be analyzed in this paper.

The present paper is organized as follows. The discussion about reflectometry methods including traditional and sequence TDR is analyzed in Section II. The details about the implementation of STDR are outlined in Section III. Section IV presents the results obtained from computational simulations. Finally, the conclusions and next works are addressed in Section V.

\section{BACKGROUND}

This section discusses the theoretical concepts about traditional TDR and sequence TDR. Traditional TDR generally uses a rectangular pulse (high-peak power pulse) with highfrequency band as a test signal to be transmitted along the loop, and to perform such a test the line should be inactive (without transmission or interfering signal).

The transmission characteristics of the TDR pulse have several inherent drawbacks. A high-peak power pulse is likely to be a significant disturber to other pairs in the binder. This type of crosstalk will most certainly violate spectral compatibility standards in DSL transmission for instance [6]. A second drawback is the requirement to produce pulses at a variety of different levels and with a variety of different durations. This equates to a requirement on the test equipment to transmit signals and to receive signals with a large dynamic range. This alone makes the possibility of incorporating this type of signal into a DSL transceiver integrated circuit very questionable [7].

On the other hand, sequence TDR uses a PN sequence as a test signal and by utilizing concepts of Direct Sequence Spread 
Spectrum (DS-SS) communications, a TDR capability has been developed which may be efficiently and cost effectively integrated into a DSL transceiver for instance.

\section{A. Traditional TDR}

TDR is widely used in the deployment of DSL services. When the line is provisioned, it is one of several test measurements used to ascertain the characteristics, topology and ultimately the capacity of the line. A measurement or reflectogram is obtained by connecting the test equipment to the line and bursting a high power pulse onto the line and measuring the reflections (echoes). Those reflections results from impedance mismatches on the line. The amount of the incident signal that is reflected toward the transmitter is determined by the reflection coefficient $\rho$ given by

$$
\rho=\frac{Z_{2}-Z_{1}}{Z_{2}+Z_{1}}
$$

where $Z_{1}$ is the impedance looking into the line from the transmitter and $Z_{2}$ is the impedance looking into the anomaly. By analyzing the position and shape of the echoes, a number of characteristics about the line can be determined including the distance to opens/shorts, the location and length of bridged taps or gauge changes, as well as the total loop length [1].

The distance to the fault is computed according to

$$
d=\frac{T_{p} \cdot V O P}{2}
$$

where $T_{p}$ is the round trip propagation time to the fault and back and $V O P$ is the velocity of propagation on the twisted pair. This term is typically about two-thirds the speed of light, but, the exact value depends on the physical wire.

Using this basic methodology, other loop attributes can be detected and analyzed. For example, a bridged tap terminated with an open will exhibit a first echo resembling a short and a second echo resembling an open. The first echo has negative polarity because the bridged tap is in parallel with the remaining portion of the line. The combined impedance is less than that of the line without the bridged tap and so the reflection coefficient is negative. A positive echo is reflected from the open at the end of the bridged tap. The length of the bridged tap can be found by computing the distance between the negative and positive echoes [1].

Implicit in (2) is the test ability of equipment to estimate the propagation time $T_{p}$. The estimation performance is limited by interference coupled into the line from adjacent pairs in the same binder and by the dispersive nature of the line. As the distance to faults increases, both the attenuation and distortion to the pulse increases. Therefore, typical TDRs have the capability to adjust signal parameters to aid in the search for faults. The two parameters available for adjustment are the transmit level of the pulse and the duration of the pulse. A narrow pulse is generally preferred since this directly impacts the resolvability of closely spaced faults. But, this is only effective at short distances. At larger distances, increasing the signal level and pulse duration is needed to produce sufficient energy for good detectability. Also, the types of disturbers present in the binder may necessitate modifying the transmit signal [3].

\section{B. Spread Spectrum and Sequence TDR}

Some concepts on spread spectrum TDR [5], [8], [9] as well as sequence TDR [4], [7] are discussed now. It is worth pointing out that STDR is the main objective, however SSTDR is briefly cited for convenience. Those two reflectometry techniques use spread spectrum modulation to transmit a test signal $s(t)$ (PN sequence) over the line. Besides spread spectrum modulation, SSTDR also uses PSK (Phase Shift Keying) modulation to create the transmitted signal. On the other hand, STDR uses only the PN signal as the transmitted signal. A schematic setup for SSTDR and STDR circuits is depicted in Figure 1.

The schematic for S/SSTDR implementation shown in Figure 1 creates first a specific kind of PN code (length, frequency, amplitude, etc), depending on the nature of the test, depicted in point $A$ at Figure. This code may be a M-Sequence Code (Maximal Length Sequence), a Gold Code, or a pair of complementary codes such as Golay Codes [10], [11], [12], [13]. Next, this signal is injected onto the PN Sequence block to generate the waveform of PN sequence $s(t)$ indicated in point $B$. This signal $s(t)$ is transmitted along the cable in case of sequence TDR. The BPSK block was not implemented yet, thus only sequence TDR may be performed. The total signal from the loop named by $s^{\prime}(t)$ in point $D$, including any noise signals and reflections from impedance discontinuities in the cable, is fed into the Correlator block along with a delayed copy of the transmitted signal $s(t+\tau)$. This delayed signal $s(t+\tau)$ (point $C$ ) is obtained in Time Shift block by adding a number of zeros in the signal $s(t)$. In the Correlator block, the cross-correlation between the received signal $s^{\prime}(t)$ (point $D$ ) and the delayed signal $s(t+\tau)$ (point $C$ ) is performed (point $E$ ). Mathematically, this cross-correlation is defined as it follows

$$
R_{s s^{\prime}}(\tau)=\frac{1}{K T_{c}} \int_{0}^{K T_{c}} s(t+\tau) s^{\prime}(t) d \tau
$$

where $T_{c}$ is the chip duration of the PN sequence, $K$ is the length of the PN sequence, and $K T_{c}$ is the PN sequence duration in seconds. Thus, the last step is to plot the STDR trace in time domain; STDR Trace block carries out that curve.

The location of the various peaks in the full correlation $R_{s s^{\prime}}(\tau)$, defined in (3), indicates the location of impedance discontinuities such as open circuits, short circuits, bridged taps and gauge changes along the loop.

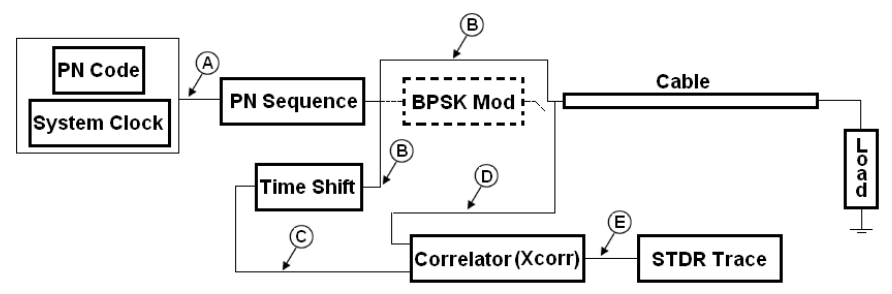

Fig. 1. S/SSTDR schematic setup. 
Spread spectrum signals, both in baseband (STDR) and modulated (SSTDR), are detectable through cross-correlation, regardless the presence of noise, other digital signals and reflections on the line [5], [8], [9]. The ability to pick out the signal is due to processing gain $L_{c}$ defined as it follows

$$
L_{c}=\frac{T_{b}}{T_{c}}=\frac{R_{c}}{R_{b}}=\frac{W}{R_{b}}
$$

where $W$ is the bandwidth of the spread-spectrum signal, $T_{b}$ denotes the duration of one entire S/SSTDR sequence (considering the entire sequence equal to one bit in communicationsystem terms), $R_{c}$ represents the chip rate of the PN sequence in chips per second, and $R_{b}$ is the bit rate, which in this case is the number of full sequences per second [8].

\section{STDR IMPLEMENTATION}

This section describes algorithm implementation for performing sequence TDR in telephone loops used for xDSL transmission. First, methods for generating PN sequences are discussed; Golay codes (complementary codes) and Maximum-Length codes (M-sequences) are briefly outlined. Next, the methods for performing correlation, auto-correlation and cross correlation, are described.

\section{A. PN Sequences}

Depending on the application, the PN sequences may be a crucial factor for limiting or not the method. To define which PN sequence is the best for a specific application, many variables can be taken into account. Correlation, code implementation, hardware implementation and time processing are some of them. We are focused in discussing correlation properties and code implementation of the PN sequences.

The basic idea of sequence TDR is to use a signal with a sufficiently narrow auto-correlation function, similar to the Dyrac's delta, rather than a short duration signal itself (highfrequency pulse); the response resolution of the sequence TDR is determined by the width of the auto-correlation function of a probe signal which may be long and with a large duty cycle [11].

The optimal PN sequence depends on the nature of the application. In our application, the best PN sequence should have a narrow main lobe with a high peak and to present the lowest side lobes in its auto-correlation. It is worth emphasizing that such a PN sequence is restricted for applications where only one PN sequence will be used at a time, that is the test is performed in only one cable; without adjacent interfering lines [8]. To obtain such a PN sequence with a single autocorrelation peak and the lack of side lobes, two kinds of code may be used: M-sequences and Golay codes [11], [10]. Figure 2 shows the waveforms of a pair of complementary PN sequences (a pair of Golay code of length $K=128$ ) transmitted into the line: $P N A$ and $P N B$. The received signals from the line, Received $A$ and Received $B$, is also shown. The auto-correlation of interest identified by Code $C$ is obtained by adding auto-correlation of Code $A$ to the auto-correlation of Code $B$ [11], [10]; this sum allows to obtain an auto-correlation without side lobes as depicted in Figure 3.

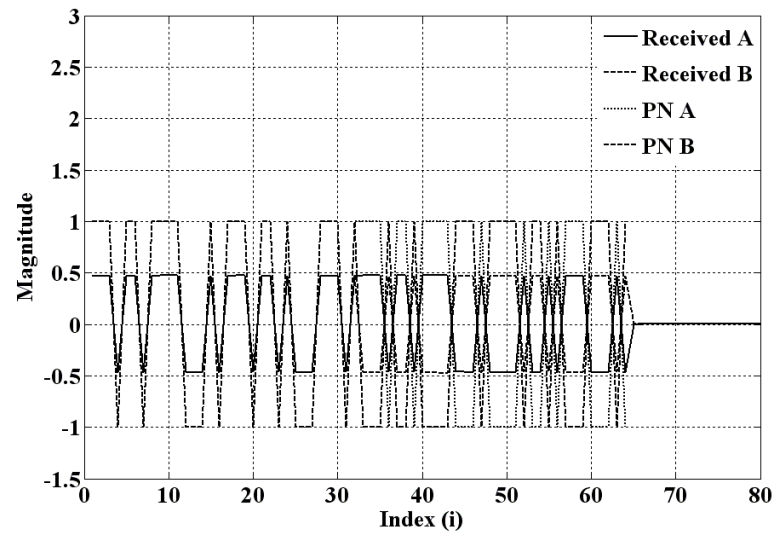

Fig. 2. Waveforms of Transmitted and Received PN sequences from the line.

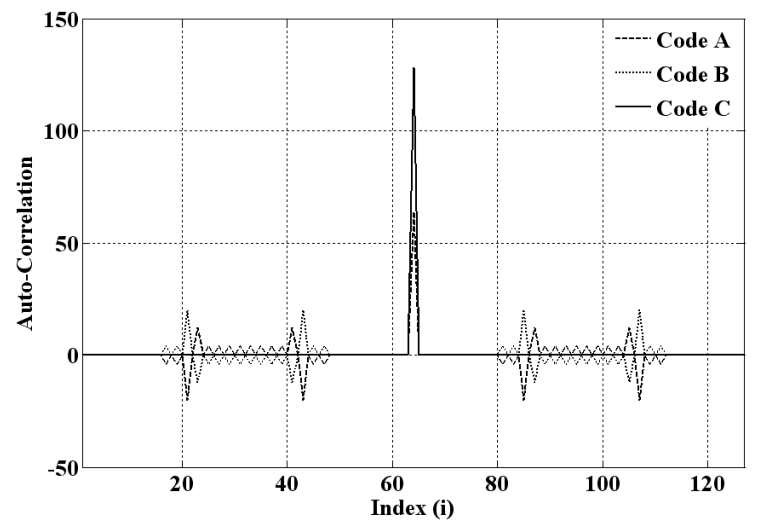

Fig. 3. Auto-correlation function of complementary codes A and $\mathrm{B}(C=$ $A+B)$ ).

\section{B. Correlation}

Sequence TDR may be performed by using two kinds of test signals. One of them utilizes a high-peak auto-correlation as a test signal to be injected into the line. The auto-correlation function may be defined as it follows in equation (3); however, the correlation is carried out by using $s(t)$ only instead of $s^{\prime}(t)$ and $s(t)$.

In that case, the PN sequence is used only for obtaining the auto-correlation with desired characteristics. This method is very simple to be code implemented, on the other hand, the hardware implementation limits its application for telephone lines carrying xDSL signals. The reason for such a limitation comes from the fact that is too difficult to generate that periodic high-frequency signal.

The second method for performing sequence TDR is to utilize PN sequence as a test signal to be transmitted down the cable. After transmission, the received PN signal is correlated with a delayed copy of transmitted PN signal, thus the correlator output may be analyzed for locating impedance mismatches in the cable. Those two methods are outlined next.

1) Auto-Correlation Method: (ACM): This method for performing STDR utilizes an auto-correlation function as a test signal as described before. This signal is transmitted into the 
line, thus the TDR trace may be obtained by convolution of echo impulse response $h(t)$ of the line and the auto-correlation function of the PN sequence as shown below

$$
\begin{aligned}
\operatorname{STDR}_{A C M}(t) & =s(t) \oplus s^{\prime}(t) \\
& =s(t) \oplus[s(t) \otimes h(t)] \\
& =[s(t) \oplus s(t)] \otimes h(t)
\end{aligned}
$$

where $\oplus$ denotes correlation function, $\otimes$ denotes convolution in time domain, $s(t)$ is the PN sequence transmitted into the line, $h(t)$ is the echo impulse response of the line, and $s^{\prime}(t)$ is the received signal from the line which is equal to the convolution between $h(t)$ and $s(t)$.

Using associative property of convolution, then it enables to calculate the auto-correlation function of the PN sequence before transmitting it into the line. This procedure has the same effect of transmitting the auto-correlation function as a test signal into the line as defined in (5).

This method is quite simple to be code implemented, however it limits the implementation in a real xDSL transceiver. This implementation would require a high-clock system to generate the high-peak auto-correlation function as a test signal.

2) Cross-Correlation Method: (CCM): Although this method provides the same result of the method which utilizes the auto-correlation function as a test signal, this method of performing STDR is quite simple to be implemented in a real xDSL transceiver. This method requires less hardware complexity because transmitting a high-frequency digital data into the line is easier than transmitting a high-peak autocorrelation function.

The implementation consists, first, in generating a PN sequence with the characteristics discussed in preview sections. Then, such a PN sequence $s(t)$ is probed into the line by mean of convolution with echo impulse response $h(t)$; thus the PN sequence is the test signal. The reflected signal $s^{\prime}(t)$ from the line is equal to the convolution $s^{\prime}(t)=s(t) \otimes h(t)$ as discussed before. The difference between the two methods comes from the fact that the correlation, performed after transmission, in this case is a cross-correlation function. It is worth emphasizing that spread-spectrum technique is applied at the transmitter for obtaining the TDR trace; the received signal from the loop $s^{\prime}(t)=s(t) \otimes h(t)$ is then correlated with a delayed copy of the transmitted signal $s(t+\tau)$ as it follows in equation below.

$$
\begin{aligned}
\operatorname{STDR}_{C C M}(t) & =s(t+\tau) \oplus s^{\prime}(t) \\
& =s(t+\tau) \oplus[s(t) \otimes h(t)]
\end{aligned}
$$

\section{Simulated Results}

The first stage consists in simulating STDR in a inactive short-loops without noise or interfering signal with lengths equal to $200 \mathrm{~m}, 150 \mathrm{~m}, 100 \mathrm{~m}, 75 \mathrm{~m}, 50 \mathrm{~m}$, and $25 \mathrm{~m}$. The second stage consists in simulating STDR in a inactive shortloops presenting mixture of gauges $(0.40 \mathrm{~mm}$ and $0.50 \mathrm{~mm})$. The last stage consists in simulating STDR in a inactive short-loops presenting one bridged tap (50m-length) with its location varying along the line (at the beginning, at the center, and at the end of the line).

Tables I, II, and III summarize loop topologies tested in this application where $L, S$, and $B T$ stand for loop, segment, and bridged tap respectively.

\section{A. Test Loop 1}

Table IV shows the results obtained from computational simulation of STDR, using the test signal depicted in Figure 2, as well as traditional TDR (Pulse Width $=100 \mathrm{~ns}$ ). In those simulations, both methods of STDR (ACM and CCM) provided the same results for calculating the total loop length. In this case, only single loops, i.e. loops with one segment, were tested.

\section{B. Test Loop 2}

Table V summarizes the results for STDR and traditional TDR (Pulse Width $=100 \mathrm{~ns}$ ) applied to telephone loops with two kinds of gauge $(0.40 \mathrm{~mm}$ and $0.50 \mathrm{~mm})$. Also, Figures 4 (loop composed by two serial segments, first $50 \mathrm{~m}$-length with $0.40 \mathrm{~mm}$ gauge, and second $150 \mathrm{~m}$-length with $0.50 \mathrm{~mm}$ ) and 5 (loop composed by two serial segments, first $50 \mathrm{~m}$-length with $0.50 \mathrm{~mm}$ gauge, and second $150 \mathrm{~m}$-length with $0.40 \mathrm{~mm}$ ) present the TDR traces comparing STDR, using ACM and CCM, to traditional TDR.

TABLE I

TOPOLOGIES FOR TEST LOOP 1.

\begin{tabular}{|c|c|c|c|c|c|c|}
\hline Gauge & L1 & L2 & L3 & L4 & L5 & L6 \\
\hline $0.40 \mathrm{~mm}$ & $25 \mathrm{~m}$ & $50 \mathrm{~m}$ & $75 \mathrm{~m}$ & $100 \mathrm{~m}$ & $150 \mathrm{~m}$ & $200 \mathrm{~m}$ \\
\hline $0.50 \mathrm{~mm}$ & $25 \mathrm{~m}$ & $50 \mathrm{~m}$ & $75 \mathrm{~m}$ & $100 \mathrm{~m}$ & $150 \mathrm{~m}$ & $200 \mathrm{~m}$ \\
\hline
\end{tabular}

TABLE II

TOPOLOGIES FOR TEST LOOP 2.

\begin{tabular}{|c|c|c|}
\hline Loop & S1 (gauge/length) & S2 (gauge/length) \\
\hline L1 & $0.40 \mathrm{~mm} / 050 \mathrm{~m}$ & $0.50 \mathrm{~mm} / 150 \mathrm{~m}$ \\
\hline L2 & $0.40 \mathrm{~mm} / 100 \mathrm{~m}$ & $0.50 \mathrm{~mm} / 100 \mathrm{~m}$ \\
\hline L3 & $0.40 \mathrm{~mm} / 150 \mathrm{~m}$ & $0.50 \mathrm{~mm} / 050 \mathrm{~m}$ \\
\hline L4 & $0.50 \mathrm{~mm} / 050 \mathrm{~m}$ & $0.40 \mathrm{~mm} / 150 \mathrm{~m}$ \\
\hline L5 & $0.50 \mathrm{~mm} / 100 \mathrm{~m}$ & $0.40 \mathrm{~mm} / 100 \mathrm{~m}$ \\
\hline L6 & $0.50 \mathrm{~mm} / 150 \mathrm{~m}$ & $0.40 \mathrm{~mm} / 050 \mathrm{~m}$ \\
\hline
\end{tabular}

TABLE III

TOPOLOGIES FOR TEST LOOP 3.

\begin{tabular}{|c|c|c|c|}
\hline Loop & S1 (gauge/length) & BT (gauge) & S2 (gauge/length) \\
\hline L1 & $0.40 \mathrm{~mm} / 050 \mathrm{~m}$ & $0.40 \mathrm{~mm}$ & $0.40 \mathrm{~mm} / 150 \mathrm{~m}$ \\
\hline L2 & $0.40 \mathrm{~mm} / 100 \mathrm{~m}$ & $0.40 \mathrm{~mm}$ & $0.40 \mathrm{~mm} / 100 \mathrm{~m}$ \\
\hline L3 & $0.40 \mathrm{~mm} / 150 \mathrm{~m}$ & $0.40 \mathrm{~mm}$ & $0.40 \mathrm{~mm} / 050 \mathrm{~m}$ \\
\hline L4 & $0.50 \mathrm{~mm} / 050 \mathrm{~m}$ & $0.50 \mathrm{~mm}$ & $0.50 \mathrm{~mm} / 150 \mathrm{~m}$ \\
\hline L5 & $0.50 \mathrm{~mm} / 100 \mathrm{~m}$ & $0.50 \mathrm{~mm}$ & $0.50 \mathrm{~mm} / 100 \mathrm{~m}$ \\
\hline L6 & $0.50 \mathrm{~mm} / 150 \mathrm{~m}$ & $0.50 \mathrm{~mm}$ & $0.50 \mathrm{~mm} / 050 \mathrm{~m}$ \\
\hline
\end{tabular}


TABLE IV

RESULTS FOR TEST LOOP 1.

\begin{tabular}{|c|c|c|}
\hline Loop 1 $(25 \mathrm{~m})$ & Gauge $0.40 \mathrm{~mm}$ & Gauge $0.50 \mathrm{~mm}$ \\
\hline STDR & $24.70 \mathrm{~m}$ & $24.83 \mathrm{~m}$ \\
\hline TDR & $29.64 \mathrm{~m}$ & $29.79 \mathrm{~m}$ \\
\hline Loop 2 $(50 \mathrm{~m})$ & Gauge $0.40 \mathrm{~mm}$ & Gauge $0.50 \mathrm{~mm}$ \\
\hline STDR & $49.56 \mathrm{~m}$ & $49.65 \mathrm{~m}$ \\
\hline TDR & $54.33 \mathrm{~m}$ & $54.28 \mathrm{~m}$ \\
\hline Loop 3 $(75 \mathrm{~m})$ & Gauge $0.40 \mathrm{~mm}$ & Gauge $0.50 \mathrm{~mm}$ \\
\hline STDR & $74.75 \mathrm{~m}$ & $74.81 \mathrm{~m}$ \\
\hline TDR & $79.03 \mathrm{~m}$ & $79.27 \mathrm{~m}$ \\
\hline Loop 4 $(100 \mathrm{~m})$ & Gauge $0.40 \mathrm{~mm}$ & Gauge $0.50 \mathrm{~mm}$ \\
\hline STDR & $100.1 \mathrm{~m}$ & $100.1 \mathrm{~m}$ \\
\hline TDR & $104.1 \mathrm{~m}$ & $104.3 \mathrm{~m}$ \\
\hline Loop 5 $(150 \mathrm{~m})$ & Gauge $0.40 \mathrm{~mm}$ & Gauge $0.50 \mathrm{~mm}$ \\
\hline STDR & $149.6 \mathrm{~m}$ & $149.6 \mathrm{~m}$ \\
\hline TDR & $153.0 \mathrm{~m}$ & $153.2 \mathrm{~m}$ \\
\hline Loop 6 $(200 \mathrm{~m})$ & Gauge $0.40 \mathrm{~mm}$ & Gauge $0.50 \mathrm{~mm}$ \\
\hline STDR & $201.2 \mathrm{~m}$ & $199.4 \mathrm{~m}$ \\
\hline TDR & $204.3 \mathrm{~m}$ & $202.5 \mathrm{~m}$ \\
\hline
\end{tabular}

It is clear in the graphics a better STDR performance than traditional TDR for calculating the total loop length and locating the gauge change (positive and negative). Both methods of STDR (ACM and CCM) calculate the total loop length and the gauge change at $203.4 m(\mid$ error $\mid=1.7 \%)$ and $49.82 \mathrm{~m}$ (|error $\mid=0.36 \%$ ) respectively for the loop in Figure 4. In this loop, traditional TDR calculated the total loop length equals to $206.5 \mathrm{~m}$ ( $\mid$ error $\mid=3.25 \%$ ) while the the gauge change was located at $54.62 m(\mid$ error $\mid=9.24 \%)$. The reults for the loop in Figure 5 followed the same fashion as the loop in Figure 4; additional informations may be obtained in Table $\mathrm{V}$ which summarizes all the results for loops with mixture of gauges.

\section{Test Loop 3}

Table VI summarizes the results for STDR and traditional TDR (Pulse Width = 100ns) applied to telephone loops with one bridged tap. Also, Figures 6 (200m-length loop $(0.40 \mathrm{~mm})$ and one $50 \mathrm{~m}$-length bridged tap $(0.40 \mathrm{~mm})$ positioned at $50 \mathrm{~m}$ from the source) and $7(200 \mathrm{~m}$-length loop $(0.50 \mathrm{~mm})$ and one $50 \mathrm{~m}$-length bridged tap $(0.50 \mathrm{~mm})$ positioned at $150 \mathrm{~m}$ from the source) present the TDR traces comparing STDR to traditional TDR.

In Figure 6, STDR test detected the first negative reflection in $49.82 \mu \mathrm{s}$ whereas TDR detected in $54.45 \mu \mathrm{s}$; therefore STDR located the bridged tap at $49.82 m(\mid$ error $\mid=0.36 \%)$ while TDR did it at $54.45 m(\mid$ error $\mid=8.9 \%)$. The first positive reflection was detected at $100.6 \mu \mathrm{s}$ and at $104.6 \mu \mathrm{s}$ by STDR and TDR tests respectively; thus the bridged tap length, calculated by STDR and TDR tests respectively, is equal to $50.78 m(\mid$ error $\mid=1.56 \%)$ and $50.15 m(\mid$ error $\mid=0.30 \%)$. The second negative reflection presented in that result is caused by the forward and backward travelling reflection from the bridged tap attachment; in this case, such a reflection will be treated as a spurious reflection. The total loop length is calculated by using the second positive reflection; STDR test determined it at $204.7 \mathrm{~m}$ (|error $\mid=2.35 \%$ ) against $207.9 \mathrm{~m}$ $(\mid$ error $\mid=3.95 \%)$ provided by TDR. The same procedure may be applied to Figure 7, on the other hand, special attention

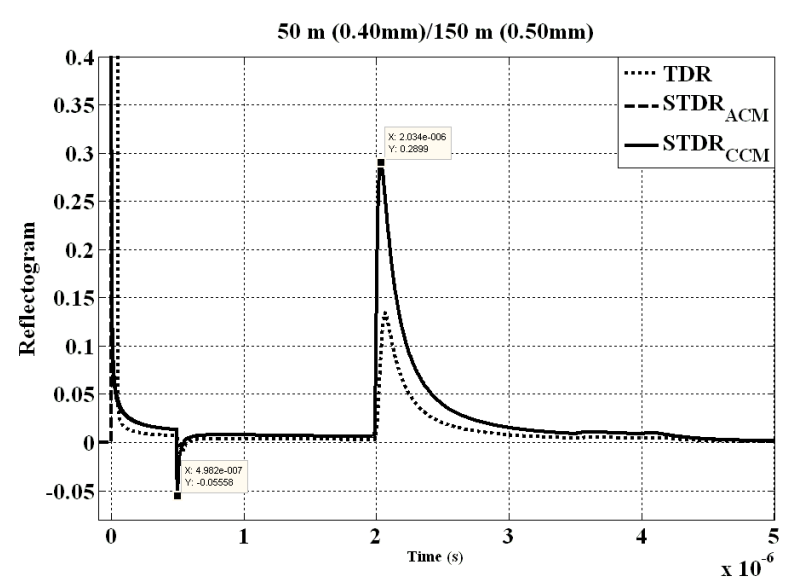

Fig. 4. STDR versus traditional TDR for $50 \mathrm{~m} / 0.40 \mathrm{~mm}+150 \mathrm{~m} / 0.50 \mathrm{~mm}$.

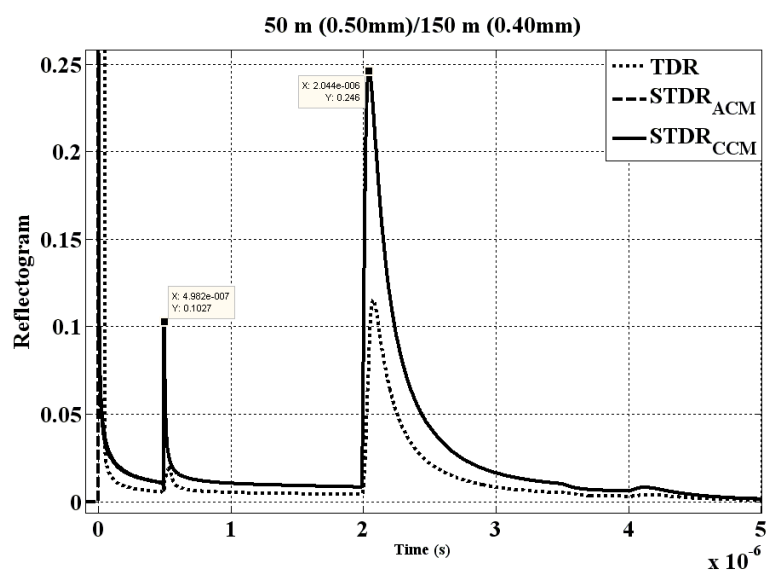

Fig. 5. STDR versus traditional TDR for $50 \mathrm{~m} / 0.50 \mathrm{~mm}+150 \mathrm{~m} / 0.40 \mathrm{~mm}$.

should be given to the second, positive, reflection which, in this case, is the sum of the reflection caused by the end of the loop and the end of the bridged tap. The reason for that is the bridged tap is located almost at the end of the loop. Additionaly, the length segment after the bridged tap is calculated by the second positive reflection; that is subtracting the first positive reflection from the second positive reflection.

\section{Conclusions}

This paper presented the usage of a new method, sequence time domain reflectometry, to perform reflectometry applied to subscriber telephone loops. This method shown to be feasible for performing efficiently reflectometry; it provides high accurate results when applied to very short loops $(\leq 200 \mathrm{~m})$, even though with mixture of gauges and bridged taps, compared to traditional TDR. Additionally, sequence TDR enables the implementation of reflectometry tests in DSL transceiver then no costly measuring equipment is required.

Next, this research will extend the application of sequence TDR for longer loops in presence of noise and transmitting DSL signals (active loops). Additionally, spread spectrum time domain reflectometry implementation is one of the next goal of this research. 
TABLE V

RESULTS FOR TEST LOOP 2.

\begin{tabular}{|c|c|c|c|c|c|c|}
\hline Loop & S1(Gauge/Length) & STDR (Length) & TDR (Length) & Total Length & STDR & TDR \\
\hline Loop 1 & $0.40 \mathrm{~mm} / 050 \mathrm{~m}$ & $49.82 \mathrm{~m}$ & $54.62 \mathrm{~m}$ & $200 \mathrm{~m}$ & $203.4 \mathrm{~m}$ & $206.5 \mathrm{~m}$ \\
\hline Loop 2 & $0.40 \mathrm{~mm} / 100 \mathrm{~m}$ & $101.0 \mathrm{~m}$ & $104.8 \mathrm{~m}$ & $200 \mathrm{~m}$ & $203.7 \mathrm{~m}$ & $207.0 \mathrm{~m}$ \\
\hline Loop 3 & $0.40 \mathrm{~mm} / 150 \mathrm{~m}$ & $152.9 \mathrm{~m}$ & $156.2 \mathrm{~m}$ & $200 \mathrm{~m}$ & $204.4 \mathrm{~m}$ & $207.5 \mathrm{~m}$ \\
\hline Loop 4 & $0.50 \mathrm{~mm} / 050 \mathrm{~m}$ & $49.82 \mathrm{~m}$ & $54.45 \mathrm{~m}$ & $200 \mathrm{~m}$ & $204.4 \mathrm{~m}$ & $207.5 \mathrm{~m}$ \\
\hline Loop 5 & $0.50 \mathrm{~mm} / 100 \mathrm{~m}$ & $100.1 \mathrm{~m}$ & $104.4 \mathrm{~m}$ & $200 \mathrm{~m}$ & $203.7 \mathrm{~m}$ & $207.0 \mathrm{~m}$ \\
\hline Loop 6 & $0.50 \mathrm{~mm} / 200 \mathrm{~m}$ & $151.4 \mathrm{~m}$ & $155.1 \mathrm{~m}$ & $200 \mathrm{~m}$ & $203.4 \mathrm{~m}$ & $206.5 \mathrm{~m}$ \\
\hline
\end{tabular}

TABLE VI

RESULTS FOR FOR TEST LOOP 3

\begin{tabular}{|c|c|c|c|c|c|c|}
\hline Loop & L1 & L2 & L3 & L4 & L5 & L6 \\
\hline S1 & $50 \mathrm{~m} / 0.40 \mathrm{~mm}$ & $100 \mathrm{~m} / 0.40 \mathrm{~mm}$ & $150 \mathrm{~m} / 0.40 \mathrm{~mm}$ & $50 \mathrm{~m} / 0.50 \mathrm{~mm}$ & $100 \mathrm{~m} / 0.50 \mathrm{~mm}$ & $150 \mathrm{~m} / 0.50 \mathrm{~mm}$ \\
\hline STDR & $49.82 \mathrm{~m}$ & $100.6 \mathrm{~m}$ & $152.3 \mathrm{~m}$ & $49.65 \mathrm{~m}$ & $100.1 \mathrm{~m}$ & $151.1 \mathrm{~m}$ \\
\hline TDR & $54.45 \mathrm{~m}$ & $104.6 \mathrm{~m}$ & $155.7 \mathrm{~m}$ & $54.45 \mathrm{~m}$ & $104.3 \mathrm{~m}$ & $154.7 \mathrm{~m}$ \\
\hline BT & $50 \mathrm{~m} / 0.40 \mathrm{~mm}$ & $50 \mathrm{~m} / 0.40 \mathrm{~mm}$ & $50 \mathrm{~m} / 0.40 \mathrm{~mm}$ & $50 \mathrm{~m} / 0.50 \mathrm{~mm}$ & $50 \mathrm{~m} / 0.50 \mathrm{~mm}$ & $50 \mathrm{~m} / 0.50 \mathrm{~mm}$ \\
\hline STDR & $50.78 \mathrm{~m}$ & $51.70 \mathrm{~m}$ & $52.60 \mathrm{~m}$ & $50.45 \mathrm{~m}$ & $51.00 \mathrm{~m}$ & $51.60 \mathrm{~m}$ \\
\hline TDR & $50.15 \mathrm{~m}$ & $51.10 \mathrm{~m}$ & $52.30 \mathrm{~m}$ & $49.85 \mathrm{~m}$ & $50.40 \mathrm{~m}$ & $51.30 \mathrm{~m}$ \\
\hline Length & $200 \mathrm{~m}$ & $200 \mathrm{~m}$ & $200 \mathrm{~m}$ & $200 \mathrm{~m}$ & $200 \mathrm{~m}$ & $200 \mathrm{~m}$ \\
\hline STDR & $204.7 \mathrm{~m}$ & $204.7 \mathrm{~m}$ & $204.4 \mathrm{~m}$ & $202.7 \mathrm{~m}$ & $202.1 \mathrm{~m}$ & $203.1 \mathrm{~m}$ \\
\hline TDR & $207.9 \mathrm{~m}$ & $207.7 \mathrm{~m}$ & $207.5 \mathrm{~m}$ & $206.0 \mathrm{~m}$ & $205.9 \mathrm{~m}$ & $206.4 \mathrm{~m}$ \\
\hline
\end{tabular}

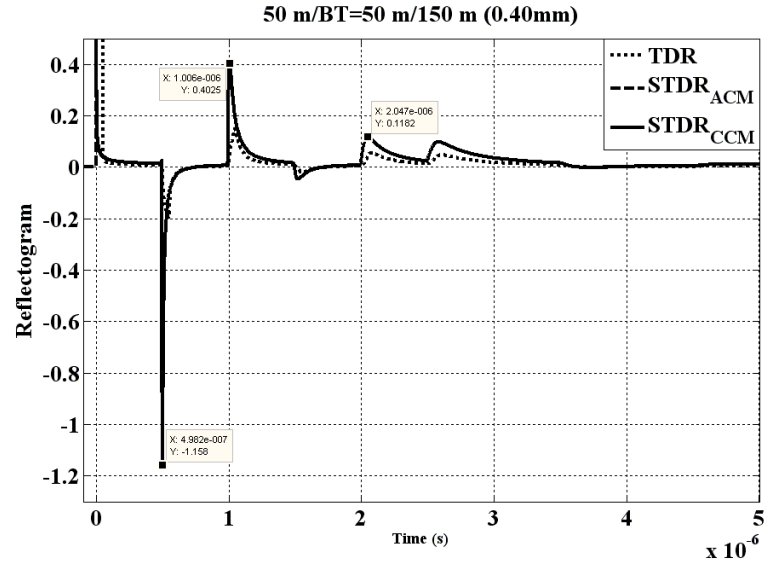

Fig. 6. STDR versus traditional TDR for $0.40 \mathrm{~mm}$-loop with one bridged tap.

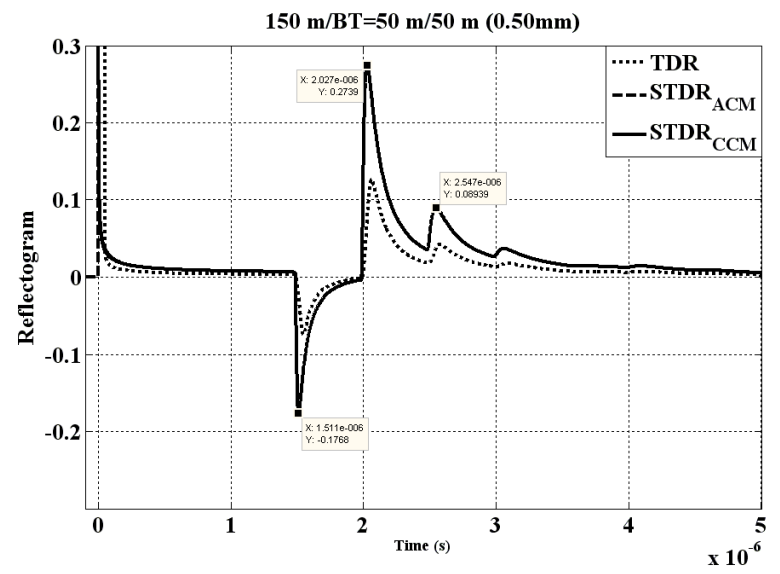

Fig. 7. STDR versus traditional TDR for $0.50 \mathrm{~mm}$-loop with one bridged tap.

\section{ACKNOWLEDGMENTS}

Some authors wish to acknowledge the support received from the Swedish Agency for Innovation Systems, VINNOVA, and the CELTIC Initiative within the EUREKA program, which partially enabled this work.

\section{REFERENCES}

[1] S. Galli and D. L. Waring, "Loop Make-up Identification via Single Ended Testing: Beyond Mere Loop Qualification," IEEE Journal on Selected Areas in Communications, vol. 20, pp. 923-935, June 2002.

[2] S. Galli and K. J. Kerpez, "Single-Ended Loop Make-up Identification Part I: A Method of Analyzing TDR Measurements," IEEE Transactions on Instrumentation and Measurements, vol. 55, pp. 528-537, April 2006.

[3] K. J. Kerpez and S. Galli, "Single-Ended Loop Make-up Identification Part II: Improved Algorithms and Performance Results," IEEE Transactions on Instrumentation and Measurements, vol. 55, pp. 538-549, April 2006.

[4] C.R. Sharma and C. Furse and R.R. Harrison, "Low-Power STDR CMOS Sensor for Locating Faults in Aging Aircraft Wiring," IEEE Sensors Journal, vol. 7, pp. 43-50, January 2007.

[5] P. Smith, "Spread Spectrum Time Domain Reflectometry," Ph.D. dissertation, Utah State University, 2003.

[6] International Telecommunications Union - ITU, "Test Procedures for DSL Transceivers Standard - G.996.1," 1999.

[7] K. Jones and G.I.S. Trevio and W.W. Jones and R.H. Jonsson, "Adaptive Method and Apparatus for Transmission Line Analysis," 2002.

[8] P. Smith, C. Furse, and J. Gunther, "Analysis of Spread Spectrum Time Domain Reflectometry for Wire Fault Location," IEEE Sensors Journal, vol. 5, pp. 1469-1478, December 2005.

[9] C. Furse, P. Smith, M. Safavi, and C. Lo, "Feasibility of Spread Spectrum Sensors for Location of Arcs on Live Wires," IEEE Sensors Journal, vol. 5, pp. 1445-1450, December 2005.

[10] D. Sawarte and M. Pursley, "Crosscorrelation Properties of Pseudorandom and Related Sequences," Proceedings of The IEEE, vol. 68, pp. 593-619, May 1980.

[11] F. Mendieta, A. Trevino, and C. Martinez, "Complementary Sequence Correlations with Applications to Reflectometry Studies," Journal of The Mexican Society of Instrumentation, vol. 3, pp. 37-46, April 1996.

[12] S. Haykin, Communication Systems, 4th ed. Wiley, 1999.

[13] J. G. Proakis, Digital Communications, 4th ed. McGraw-Hill, 2001. 